\title{
A chewable low-dose oral contraceptive: a new birth control option?
}

\author{
This article was published in the following Dove Press journal: \\ Patient Preference and Adherence \\ 2I April 2012 \\ Number of times this article has been viewed
}

\section{Edith Weisberg ${ }^{1,2}$ \\ 'Sydney Centre for Reproductive Health Research, Research Division of Family Planning NSW, ${ }^{2}$ Department of Obstetrics and Gynaecology, Queen Elizabeth II Research Institute for Mothers and Infants, University of Sydney, Sydney, Australia}

\begin{abstract}
A new chewable combined oral contraceptive pill containing ethinyl estradiol (EE) $0.025 \mathrm{mg}$ and norethindrone (NE) $0.8 \mathrm{mg}$ in a $24 / 4$ regimen was approved for marketing in December 2010. Each of the four inactive tablets contains $75 \mathrm{mg}$ ferrous fumarate, which has no therapeutic benefit. The tablet can be taken with food but not water as this affects the absorption of EE. The Pearl index based on intention to treat women aged 18-35 years has been reported at 2.01 (confidence interval [CI] 1.21,3.14) and for the whole population 1.65 (CI 1.01, 2.55). The effect of a body mass index of $>35$ was not studied. Regular withdrawal bleeding occurred for $78.6 \%$ of women in Cycle 1, but by Cycle 13 almost half the women failed to have a withdrawal bleed. This new formulation provides an intermediate dose of an EE/NE combination that will be useful for women experiencing breakthrough bleeding on the lower-dose EE/NE pill. The convenience of a low-dose pill, which can be chewed without the need for water, will be useful to enable women who have forgotten a pill to take one whenever they remember, provided they carry it with them. The advantage of a 24/4 regimen is better suppression of follicular development in the pill-free interval and may be beneficial for women who experience menstrual cycle-related problems, such as heavy bleeding or dysmenorrhea.
\end{abstract}

Keywords: combined oral contraceptive, low dose, ethinyl estradiol, norethindrone

\section{Introduction}

The combined oral contraceptive pill (COC) was first marketed in 1960. In the intervening 50 years, it has undergone many changes, including a reduction in dose of both the estrogen and progestin components, different administration regimens, and the development of new progestins more similar in structure to progesterone. The aim of these changes was to reduce the risk of potentially serious side effects, such as venous and arterial thrombosis and hypertension, as well as to make the COC more acceptable to women by reducing the likelihood of troublesome side effects such as nausea, vomiting, weight gain, headaches, breast tenderness, and pain, which were common with the original high-dose COCs. The dose of estrogen has been reduced from $0.1 \mathrm{mg}$ to as low as $0.02 \mathrm{mg}$. Several COCs containing estradiol instead of ethinyl estradiol (EE) have been developed, although these are not yet available in the USA. The recent introduction of a chewable COC adds another choice for women, especially those who find swallowing a pill, even if small, difficult or unacceptable.

\section{Formulation of the chewable COC}

Each active tablet of this newly released COC contains $0.025 \mathrm{mg}$ EE and $0.8 \mathrm{mg}$ norethindrone $(\mathrm{NE})$ and, in each inactive tablet, $75 \mathrm{mg}$ ferrous fumarate. EE is the 
estrogen that is used in almost all COCs currently marketed and is available in combination with $\mathrm{NE}$ in a number of different formulations. This is the first COC that combines $0.025 \mathrm{mg}$ EE with $0.8 \mathrm{mg} \mathrm{NE}$ and offers an intermediate dose of both EE and NE compared with the range now available (EE 0.02-0.05 mg and NE 0.4-1.5 mg). Each cycle pack contains 24 light green active tablets and four brown inactive (placebo) tablets. The recommended regimen is one active tablet daily for 24 days followed by one placebo tablet daily for 4 days, providing a 28-day cycle. The placebo tablets do not serve any therapeutic purpose. The tablets are to be chewed and swallowed without water at approximately the same time each day. ${ }^{1}$

\section{Pharmacology, mode of action and pharmacokinetics}

Both EE and NE have been available in a variety of doses and combinations in COCs since the 1960s. NE has weak androgenicity and estrogenicity. ${ }^{2}$ Both EE and NE are subject to first-pass metabolism after oral dosing, resulting in an absolute bioavailability of approximately $43 \%$ for EE and $64 \%$ for NE. Data from a study of 18 women taking the chewable COC indicate that maximum plasma EE and NE concentrations occur within 2 hours after oral administration. Following multiple doses of EE/NE chewable tablets, the mean maximum concentrations of EE and NE were increased by $14 \%$ and $126 \%$, respectively, when compared with levels following a single dose. Mean EE and NE exposures (area under the time-concentration curve [AUC] values) were increased by $55 \%$ and $239 \%$, respectively, as compared to single-dose administration. ${ }^{3}$

\section{Food and water effect}

Since these tablets are chewed rather than swallowed whole, a Phase III study looked at the effect of food on plasma levels of EE and NE tablets taken either after food or fasting (Figures 1 and 2). ${ }^{3}$ Taking the tablet with food did not affect EE absorption but increased NE absorption by $10 \%$. A single EE/NE tablet taken with food decreased the $\mathrm{C}_{\max }$ of $\mathrm{NE}$ by $47 \%$ and but increased the AUC by $10 \%-14 \%$ and decreased the $\mathrm{C}_{\max }$ of EE by $39 \%$, but not AUC. The US Federal Drug Administration (FDA) decided that, despite the effect of food on absorption, the tablets could be taken with or without food because these were the instructions given to subjects participating in the Phase III clinical trial. ${ }^{4}$

There was a small reduction in the $\mathrm{C}_{\max }$ for $\mathrm{EE}$ when $\mathrm{EE} / \mathrm{NE}$ tablets were chewed with $45 \mathrm{~mL}$ water compared to the $\mathrm{C}_{\max }$ when tablets were chewed without water. The mean
AUC value for $\mathrm{EE}$ and the mean $\mathrm{C}_{\max }$ and $\mathrm{AUC}$ values for $\mathrm{NE}$ followed by $45 \mathrm{~mL}$ water, compared with the $\mathrm{C}_{\max }$ when tablets were chewed and taken without water, were within $80 \%-125 \%$ of each other, indicating bioequivalence. However, the mean $\mathrm{C}_{\max }$ for $\mathrm{EE}$ was $76.5 \%$ instead of $\geq 80 \%$ when compared to levels taken without water. As taking the tablet with water affects the bioavailability of EE but not NE, it is recommended that the tablets be chewed without water. As this tablet is meant to be chewed rather than swallowed, a study to determine any possibility of oral irritation was conducted as a single-center, open-label, uncontrolled study in 54 healthy women aged 18-44. The subjects chewed one EE/NE tablet per day without water for 24 days, irrespective of food intake. No evidence of any irritation of the oral cavity was demonstrated. ${ }^{3}$

As combinations of $\mathrm{EE}$ with $\mathrm{NE}$ have been widely available for many years, one of the few assessments carried out on this chewable COC was serum sex hormone binding globulin (SHBG) concentration, which had more than doubled by day 24 of pill intake. Mean SHBG concentrations were increased by $170 \%$ from baseline. This is to be expected, as EE induces the synthesis of SHBG. SBHG concentrations had more than doubled by day 24 due to accumulation. $^{5}$

The increase in SHBG suggests that, like many other COCs, this particular formulation may be useful to treat women with moderate acne. ${ }^{6}$ SHBG binds free testosterone, which is responsible for increased sebum production in the skin resulting in acne. ${ }^{7}$

\section{Mode of action}

All combined oral contraceptives act by suppression of gonadotropins. Although the primary mechanism is inhibition of ovulation, other alterations include increases in the viscosity of cervical mucus, which impedes sperm passage into the uterus, and changes in the endometrium, which reduce the likelihood of implantation. ${ }^{4}$

\section{Overall assessment}

A literature search found no published articles relating to this new $0.25 \mathrm{mg}$ EE/0.8 mg NE COC. The only data available are from the Phase III studies submitted to the FDA in the registration application. ${ }^{1,3-6}$ A total of 1700 subjects were enrolled in a Phase III study, of whom 1677 women took at least one dose of the study drug and were included in the intent-to-treat population analysis for safety. A modified intent-to-treat population (MITT) included all subjects who received at least one dose of the study drug and were 


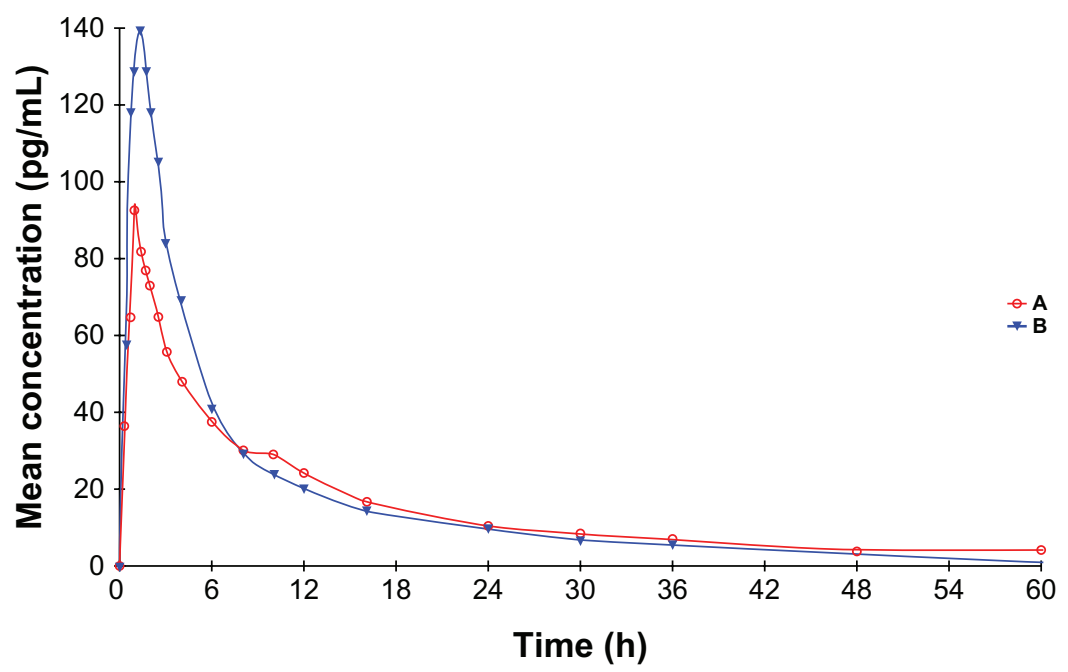

Figure I Mean plasma ethinyl estradiol concentration versus time curves following administration of a NE/EE tablet under test and reference treatments (A) fed and (B) fasted conditions, respectively, to healthy female subjects $(n=13)$.

Abbreviations: EE, ethinyl estradiol; NE, norethindrone.

evaluated for pregnancy at least once after beginning the study medication. A subgroup of the MITT population, the pregnancy intent-to-treat population (PITT), included all subjects who were 18-35 years of age at enrollment, had received at least one dose of study drug, and were evaluated for pregnancy at least once after beginning the study medication. The PITT consisted of 1251 women who were assessed over 12,297 treatment cycles during which no backup contraception was used (Table 1). ${ }^{4}$

There were 19 on-treatment pregnancies. The MITT population (a secondary efficacy population) consisted of 1570 women aged 18-46 years at entry, who were assessed over 15,752 treatment cycles during which no backup contraception was used. A single on-treatment pregnancy occurred among the subjects who were $>35$ years of age at entry. The other pregnancies all occurred in women aged $\leq 35$ years. The Pearl index ${ }^{5}$ for the PITT group was 2.01 (CI 1.21, 3.14) and for the MITT population, 1.65 (CI 1.01, 2.55). For the PITT cohort, the cumulative failure rate after 13 cycles of treatment was $2 \%$ (95\% CI 1.27\%-3.13\%), based on the 19 on-treatment pregnancies, and for the MITT group, $1.75 \%$ (CI 1.27-2.4). The Pearl index for subjects in the PITT group with a body mass index (BMI) of $\leq 30 \mathrm{~kg} / \mathrm{m}^{2}$ was 1.86 (95\% CI 1.04-3.06) compared with 2.89 (95\% CI 0.79-7.38) for subjects with a BMI between 30 and $35 \mathrm{~kg} / \mathrm{m}^{2}$, based on four pregnancies in this subgroup. The overall percent-

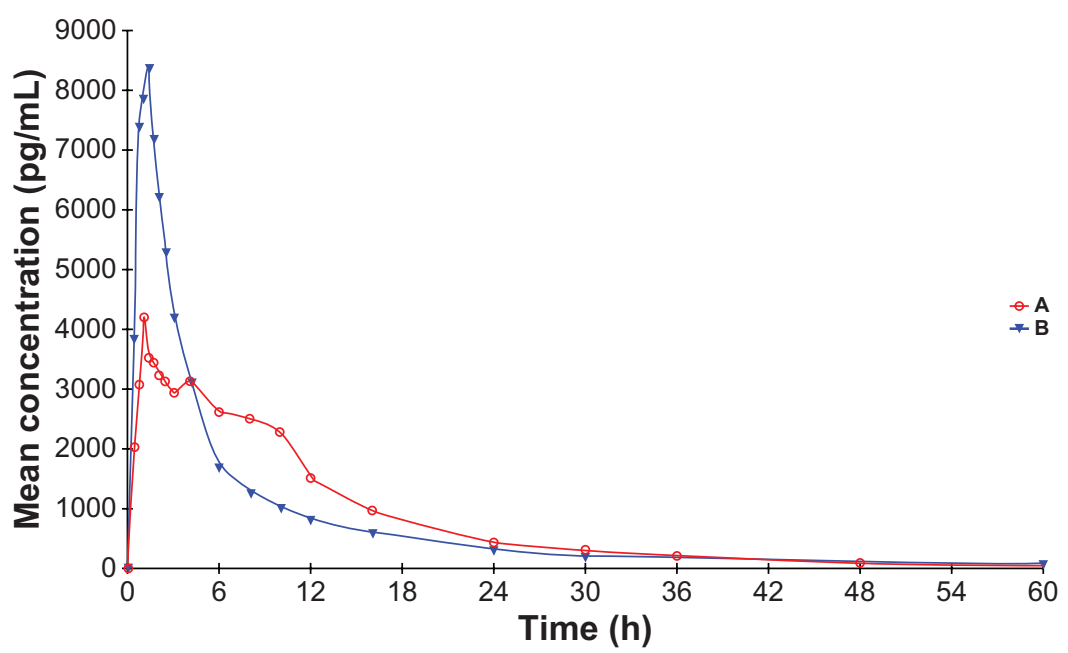

Figure 2 Mean plasma norethindrone concentration versus time curves following administration of a NE/EE tablet under test and reference treatments (A) fed and (B) fasted conditions, respectively, to healthy female subjects $(n=13)$. Abbreviations: EE, ethinyl estradiol; NE, norethindrone. 
Table I Enrollments and early withdrawals

\begin{tabular}{ll}
\hline Total subjects enrolled & 1700 \\
Total subjects treated & $1677(100 \%)$ \\
MITT population & $1570(93.6 \%)$ \\
PITT population & $1251(74.6 \%)$ \\
Prematurely discontinued from the study & $686(40.9 \%)^{\mathrm{a}}$ \\
Lost to follow-up & $271(16.2 \%)$ \\
Withdrawal of consent & $149(8.9 \%)$ \\
Adverse event E & $143(8.5 \%)$ \\
Other & $75(4.5 \%)$ \\
Protocol violation & $25(1.5 \%)$ \\
Lack of efficacy (pregnancy) & $23(1.4 \%)$ \\
\hline
\end{tabular}

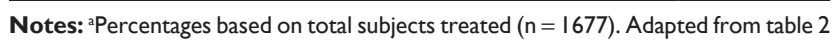
in US Center for Drug Evaluation and Research/FDA. Summary Review - Norethindrone (NE) and Ethinyl Estradiol (EE) Chewable Tablets and Ferrous Fumarate (FF) Chewable Tablets. Silver Spring, MD: FDA; 20 I0. Available from: http://www.accessdata.fda.gov/ drugsatfda_docs/nda/20 I0/0225730rig I s000SumR.pdf. Accessed February 2I, 2012. Abbreviations: MITT, modified intent to treat population; PITT, pregnancy intent to treat population.

age of subjects with BMI $\geq 30 \mathrm{~kg} / \mathrm{m}^{2}$ in the entire treated population was $263 / 1677$ (15.6\%) compared with $21 \%$ of pregnant subjects with $\mathrm{BMI} \geq 30 \mathrm{~kg} / \mathrm{m}^{2}{ }^{4}$ There is conflicting evidence on the effect of BMI on COC efficacy. ${ }^{8}$ The numbers in this Phase III study are too small to provide conclusive evidence of the effect of BMI on pregnancy rates with the chewable COC, especially as women with BMI $>35 \mathrm{~kg} / \mathrm{m}^{2}$ were excluded from the study population.

\section{Cycle control}

Cycle control is an important consideration for women in choosing a COC. In this Phase III study, regular withdrawal bleeding occurred for $78.6 \%$ of women in Cycle 1 but by Cycle 13 this had reduced to $56.6 \%$, indicating that by the final study cycle almost half the women failed to have a withdrawal bleed. There are no data available to indicate what percentage experienced more than one cycle with absent withdrawal bleeding. Breakthrough bleeding and or spotting (BTB) is common with most COCs during the initial cycles. With this COC, the percentage of cycles with BTB decreased from $31.3 \%$ in Cycle 2 to $22.5 \%$ in Cycle $13 .{ }^{4}$ The number of days with BTB decreased from 1.9 during Cycle 1 to 0.9 in Cycle 13 . Overall, approximately $70 \%$ of subjects will have some intracyclic bleeding/spotting sometime in the first year of use. A total of 15 subjects ( $0.9 \%)$ discontinued the study prematurely due to unacceptable bleeding patterns including amenorrhea.

Although there are no published studies on this formulation, the bleeding patterns are similar to those reported for an EE $0.02 \mathrm{mg} / \mathrm{NE} 0.5 \mathrm{mg}$ combination, which reported amenorrhea in $20.7 \%$ of cycles compared with $7.1 \%$ for EE $0.02 \mathrm{mg} /$ levonorgestrel $0.1 \mathrm{mg} .{ }^{9}$

\section{Overall assessment of safety}

Among the safety issues of greatest concern associated with the use of hormonal contraceptives are those related to thrombotic and thromboembolic events such as deep vein thrombosis (DVT), pulmonary emboli, and stroke. In the present study, which contained safety data from more than 1600 subjects and more than 15,500,28-day treatment cycles, there was one report of DVT and no reports of pulmonary embolus or stroke. The risk of venous thromboembolism (VTE) in women using COCs is well established and is estimated to have an incidence rate of $1 / 1000$ women. ${ }^{10}$ It usually occurs within the first 3-6 months of initiating COCs in women with hereditary thrombophilias. ${ }^{11}$ Other risk factors for VTE are smoking, immobility, increasing age, and BMI. ${ }^{12}$ A single case of DVT in the clinical development program for $\mathrm{EE} / \mathrm{NE}$ chewable tablets is not unexpected nor indicative of an increased risk of VTE. Although there is still some controversy about the risk of venous thromboembolism with the newer progestins, the consensus is that norethindrone, norethindrone acetate, and levonorgestrel are associated with the lowest risk of venous thromboembolic events. ${ }^{4}$

Apart from the single case of DVT reported in the pivotal study, eight other serious adverse events were reported that could be ascribed to this COC: depression and/or suicidal ideation (four), cholecystitis and/or cholecystectomy (two), and hypertension (two). These serious adverse events have been previously reported in association with COCs.

A total of 136 out of 1677 subjects (8.1\%) experienced an adverse event leading to early withdrawal from the study. The most frequently reported adverse events leading to discontinuation and the percentage of subjects reporting these adverse events were nausea $(1.0 \%)$, weight increase $(0.8 \%)$, acne $(0.8 \%)$, BTB $(0.7 \%)$, altered mood $(0.4 \%)$, hypertension $(0.4 \%)$, irritability $(0.3 \%)$, migraine $(0.3 \%)$, decreased libido $(0.3 \%)$, and mood swings $(0.3 \%)$. The most commonly reported adverse events $(>2 \%)$ - possibly related to treatment with the study drug and the percentage of subjects reporting them - included nausea $(6.1 \%)$, headache $(4.8 \%)$, dysmenorrhea $(3.9 \%)$, acne $(3.2 \%)$, vulvovagina mycotic infection $(3.1 \%)$, vomiting $(2.7 \%)$, increased weight $(2.3 \%)$, anxiety (2.1\%), fungal infections $(2.1 \%)$, and diarrhea (2.0\%).

Of the 1677 subjects who took study medication, 143 discontinued due to an adverse event (8.5\%). The most frequent adverse reactions leading to discontinuation were nausea $(1.0 \%)$, weight increase $(0.8 \%)$, acne $(0.8 \%)$, metrorrhagia $(0.7 \%)$, altered mood $(0.4 \%)$, hypertension $(0.4 \%)$, irritability $(0.3 \%)$, migraine $(0.3 \%)$, decreased libido $(0.3 \%)$, 
and mood swings $(0.3 \%)$. Low incidences of these side effects have been reported in virtually all hormonal contraceptive studies.

As mentioned previously, there are no reports in the literature about the $0.025 \mathrm{mg}$ EE/ $0.08 \mathrm{mg} \mathrm{NE}$ combined COC and there are no comparative studies as the only Phase III studies carried out were open-label single studies. There is another chewable COC with a similar drug combination currently approved in the USA containing $0.035 \mathrm{mg}$ $\mathrm{EE}$ and $0.4 \mathrm{mg} \mathrm{NE}$ but this is to be taken once daily for 21 days followed by one tablet of ferrous fumarate once daily for 7 days. The instructions for this COC indicate it can be can be swallowed whole or chewed with water and taken without regard to food. ${ }^{1}$ There are more than five other products with EE/NE combination approved for contraception. These products contain $0.035 \mathrm{mg} \mathrm{EE}$ or $0.05 \mathrm{mg}$ EE and either $0.5 \mathrm{mg} \mathrm{NE}$ or $1.0 \mathrm{mg} \mathrm{NE}$. As EE and $\mathrm{NE}$ have been used in much higher doses in previous COC combinations, there are no safety issues with this latest chewable COC.

Low-dose COCs tend to have increased follicular development in the pill free week compared to higher doses. ${ }^{13}$ The use of a 24/4 day regimen with low-dose formulations has generally been associated with less follicular development compared to a $21 / 7$ day regimen, ${ }^{14}$ although one study comparing a $24 / 4$ day regimen of $0.02 \mathrm{mg}$ EE/1 mg NE with a 21/7 day regimen found no difference in follicle development. ${ }^{15}$ A similar study of the same combination found that bleeding patterns were better with the 24/4 regimen with reduced intracyclic bleeding and shorter withdrawal bleeding. ${ }^{16}$ A Cochrane review was unable to detect any differences in effectiveness between COCs containing $0.02 \mathrm{mg}$ EE or $>0.02 \mathrm{mg}$ EE but found that lower doses were associated with higher rates of bleeding disturbances. ${ }^{17}$

\section{Patient acceptability}

Since its introduction in 1960, the COC has been embraced by women and is currently used by approximately 100 million women worldwide. ${ }^{8}$ There are no acceptability studies available for this new chewable COC. Compliance in the Phase III study was difficult to access, but 39 women were withdrawn for apparent failure to comply with the protocol. However, both compliance studies and acceptability studies have been carried out on other COCs. Compliance with a daily oral preparation can be difficult. A study using an electronic device that recorded actual pill use found that $30 \%$ of women missed three or more pills in any given cycle. ${ }^{18}$
In a French survey, of all unplanned pregnancies, 21\% were using $\mathrm{COC}$ at time of conception. ${ }^{19}$

As this is a newly marketed product, no uptake or continuation rates are available. In a prospective study, Rosenberg found that $37 \%$ of pill users discontinued because of side effects, the majority in the first 2 months. ${ }^{20}$ The major side effects were bleeding irregularities (12\%), nausea $(7 \%)$, weight gain $(5 \%)$, mood changes $(5 \%)$, breast tenderness $(4 \%)$, and headaches $(5 \%)$, with similar side effects to those reported with the new chewable COC. However, the incidence of these is lower with the new pill, which could be ascribed to the low dose of steroids in this formulation. The discontinuation rate for this Phase III trial was considerably lower at $6.2 \%$ but continuation rates in clinical trials are generally lower than when COCs are in general use.

\section{Conclusion}

This new chewable formulation, which was approved for sale in the USA in December 2010, offers an additional choice for women. There is only one other chewable pill on the market but this contains a higher dose of $\mathrm{EE}$ and is a $21 / 7$ regimen. The advantage of a $24 / 4$ regimen is better suppression of follicular development in the pill-free interval, reducing the likelihood of breakthrough ovulation even further. ${ }^{21}$ Reducing the active pill-free interval has been shown to be beneficial for women who experience menstrual cycle-related problems such as heavy bleeding or dysmenorrhea. The hormone-free interval of less than 7 days has additional benefits in managing hormone withdrawal symptoms such as menstrual migraine and efficacy may be improved in situations where pills are inadvertently missed or in women who are perceived as "poor" pill takers. ${ }^{22}$

The formulation of $0.025 \mathrm{mg} \mathrm{EE} / 0.4 \mathrm{mg} \mathrm{NE}$ offers an intermediate dose of these common combinations of hormones. The efficacy is comparable to other marketed COCs. The bleeding pattern is comparable to other lowerdose COCs. Some women may find the incidence of missed withdrawal bleeding stressful as they may interpret it as a sign of pregnancy. However, there are an increasing number of women who prefer to avoid menstruation. ${ }^{23}$ Careful counseling about missed withdrawal bleeds prior to commencing this COC should reassure these women.

The convenience of a low-dose pill that can be chewed without the need for water will be useful in allowing women who have forgotten a pill to take it whenever they remember, provided they carry it with them. 


\section{Disclosure}

The author declares no conflicts of interest in this work.

\section{References}

1. US Center for Drug Evaluation and Research/FDA. Highlights of Prescribing Information - Norethindrone and Ethinyl Estradiol Chewable Tablets and Ferrous Fumarate Chewable Tablets. Silver Spring, MD: FDA; nd. Available from: http://www.accessdata.fda.gov/drugsatfda_docs/ label/2010/022573s000lbl.pdf. Accessed February 21, 2012.

2. Schoonen WG, Deckers GH, de Gooijer ME, de Ries R, Kloosterboer HJ. Hormonal properties of norethisterone, 7alpha-methyl-norethisterone and their derivatives. J Steroid Biochem Mol Biol. 2000;74(4):213-222.

3. US Center for Drug Evaluation and Research/FDA. Summary ReviewNorethindrone (NE) and Ethinyl Estradiol (EE) Chewable Tablets and Ferrous Fumarate (FF) Chewable Tablets. Silver Spring, MD: FDA; 2010. Available from: http://www.accessdata.fda.gov/drugsatfda_docs/ nda/2010/022573Orig1s000SumR.pdf. Accessed February 21, 2012.

4. US Center for Drug Evaluation and Research/FDA. Clinical Pharmacology and Biopharmaceutics Review(s) - Norethindrone and Ethinyl Estradiol Chewable Tablets And Ferrous Fumarate Chewable Tablets. Silver Spring, MD: FDA; 2010. Available from: http://www.accessdata. fda.gov/drugsatfda_docs/nda/2010/022573Orig1s000ClinPharmR.pdf. Accessed February 21, 2012.

5. US Center for Drug Evaluation and Research/FDA. Medical ReviewNorethindrone (NE) and Ethinyl Estradiol (EE) Chewable Tablets and Ferrous Fumarate (FF) Chewable Tablets: NDA 22-573 (0.025 mg EE/0.8 $m g$ NE). Silver Spring, MD: FDA; 2010. Available from: http://www. accessdata.fda.gov/drugsatfda_docs/nda/2010/0225730rig1s000MedR. pdf. Accessed February 21, 2012

6. Arowojolu AO, Gallo MF, Lopez LM, Grimes DA, Garner SE. Combined oral contraceptive pills for treatment of acne. Cochrane Database Syst Rev. 2007;1:CD004425.

7. O'Connell K, Westhoff C. Pharmacology of hormonal contraceptives and acne. Cutis. 2008;81(Suppl 1):S8-S12.

8. US Center for Drug Evaluation and Research/FDA. Pharmacology Review(s) - Norethindrone and Ethinyl Estradiol Chewable Tablets and Ferrous Fumarate Chewable Tablets. Silver Spring, MD: FDA; 2010. Available from: http://www.accessdata.fda.gov/drugsatfda_ docs/nda/2010/022573Orig1s000PharmR.pdf. Accessed February 21, 2012.

9. Burkman RT, Fisher AC, Wan GJ, Barnowski CE, LaGuardia KD. Association between efficacy and body weight or body mass index for two low-dose oral contraceptives. Contraception. 2009;79(6): 424-427.
10. Endrikat J, Hite R, Bannermerschult R, Gerlinger C, Schmidt W. Multicenter, comparative study of cycle control, efficacy and tolerability of two low-dose oral contraceptives containing 20 microg. ethinylestradiol/100 microg levonorgestrel and 20 microg ethinylestradiol/500 microg norethisterone. Contraception. 2001; 64(1):3-10.

11. Gallo MF, Nanda K, Grimes DA, Lopez LM, Schulz KF. $20 \mu \mathrm{g}$ versus $>20 \mu \mathrm{g}$ estrogen combined oral contraceptives for contraception. Cochrane Database Syst Rev. 2011;1:CD003989.

12. Dinger JC, Heinemann LA, Kühl-Habich D. The safety of a drospirenonecontaining oral contraceptive: final results from the European Active Surveillance study on oral contraceptives based on 142,475 womenyears of observation. Contraception. 2007;75(5):344-354.

13. Martínez F, Avecilla A. Combined hormonal contraception and venous thromboembolism. Eur J Contracept Reprod Health Care. 2007; 12(2):97-106.

14. Anderson FA Jr, Spencer FA. Risk factors for venous thromboembolism. Circulation. 2003;107(23 Suppl 1):I9-I16.

15. Klipping C, Duijkers I, Trummer D, Marr J. Suppression of ovarian activity with a drospirenone-containing oral contraceptive in a $24 / 4$ regimen. Contraception 2008;78(1):16-25.

16. Spona J, Elstein M, Feichtinger W, et al. Shorter pill-free interval in combined oral contraceptives decreases follicular development. Contraception. 1996; 54(2):71-77.

17. Rible RD, Taylor D, Wilson ML, Stanczyk FZ, Mishell DR Jr. Follicular development in a 7-day versus 4-day hormone-free interval with an oral contraceptive containing $20 \mathrm{mcg}$ ethinyl estradiol and $1 \mathrm{mg}$ norethindrone acetate. Contraception. Mar 2009;79(3):182-188.

18. Potter L, Oakley D, de Leon-Wong E, Cañamar R. Measuring compliance among oral contraceptive users. Fam Plann Perspect 1996;28(4):154-158.

19. Bajos N, Leridon H, Goulard H, Oustry P, Job-Spira N; COCON Group. Contraception: from accessibility to efficiency. Hum Reprod. 2003;18(5):994-099.

20. Rosenberg MJ, Waugh MS. Oral contraceptive discontinuation: a prospective evaluation of frequency and reasons. Am J Obstet Gynecol. 1998;179(3 Pt 1):577-582.

21. Read CM. New regimens with combined oral contraceptive pills moving away from traditional 21/7 cycles. Eur J Contracept Reprod Health Care. 2010;15(Suppl 2):S32-S41.

22. Glasier AF, Smith KB, van der Spuy ZM, et al. Amenorrhea associated with contraception-an international study on acceptability. Contraception. 2003;67(1):1-8.

23. Fu H, Darroch JE, Haas T, Ranjit N. Contraceptive failure rates: new estimates from the 1995 National Survey of Family Growth. Fam Plann Perspect. 1999;31(2):56-63.
Patient Preference and Adherence

\section{Publish your work in this journal}

Patient Preference and Adherence is an international, peer-reviewed, open access journal focusing on the growing importance of patient preference and adherence throughout the therapeutic continuum. Patient satisfaction, acceptability, quality of life, compliance, persistence and their role in developing new therapeutic modalities and compounds to

\section{Dovepress}

optimize clinical outcomes for existing disease states are major areas of interest. This journal has been accepted for indexing on PubMed Central. The manuscript management system is completely online and includes a very quick and fair peer-review system. Visit http://www.dovepress.com/ testimonials.php to read real quotes from published authors. 